\title{
IL BOSCO IN SARDEGNA NEL MEDIOEVO
}

\author{
PINUCCIA F. SIMBULA \\ Università di Sassari
}

\begin{abstract}
E' la Sardegna un Isola del Mar Mediterraneo, la maggiore fra tutte le altre, che ritrovansi in esso doppo quella di Sicilia...Il suo continente si è più di Montagna che di Pianura. Ritrovassene di questa di assai distesa in alcune parti, in nessuna però mai a' segno di perder di vista li Monti... II Paese poi di Montagna sotto qual nome intendo anche di comprendere le Colline è generalmente più popolato, coltivo, ed ameno, che la pianura. Non è quello men fertile, che questa nella produzione dei frutti dov'è lavorato; e quella parte che non si coltiva, fornisce ottimo pascolo alla quantità grande di bestiami, che in esso si tengono.

Incontransi in una parte alberi fruttiferi di molte, e varie specie, in altra vastissimi ollivari, dove vigne, e dove folti boschi d'alberi atti al lavoro, ed altri usi necessarj, ne quali fanno la loro dimora li Cervi, li Caprioli, ed altri Animali selvatici, e dove il pastore ritrova pascolo per il suo Gregge quando già ha consonto o che l'ardor del sole ha abbrucciato quello della Campagna aperta!.
\end{abstract}

L'Anonimo piemontese nella Descrizione dell'Isola di Sardegna, a metà del Settecento, coglie uno degli elementi caratterizzanti dell'isola: la sua montuosità e il suo territorio accidentato, ricco di rilievi più o meno estesi ed elevati che predominano sulle campagne pianeggianti.

Le notizie dei cronisti arabi che si accostarono all'isola nei secoli dopo il Mille come Al Himyari e Az-Zuhri, la ricordano in qualche caso

'I. F. Fara, Opera, In Sardiniae Chorographiam, a cura di, E. Cadoni, Sassari, 1992, vol. I, p. 107. Altri riferimenti ai boschi, alla fauna $\mathrm{e}$ in generale alle risorse boschive nell'opera del Fara alle pp. 75, 115, 181, 183, 187, 189-191, 197, 199, 219-221, 223, 225, 227.

"Anuario de Estudios Medievales". 29 (1999) 
come ricca di frutti e di acqua ${ }^{2}$. Nessun esplicito riferimento ai boschi che del resto poco interessavano i viaggiatori arabi, molto più attenti alle risorse agricole. La mancanza di fonti documentarie adeguate e di studi approfonditi, unitamente alla povertà di dati di scavo, non consentono per quest'epoca di conoscere il livello della popolazione, la struttura e la continuità degli insediamenti, l'identificazione dei processi di trasformazione agraria, utili per conoscere anche la storia del bosco e della sua integrazione nell'economia'.

L'avanzata demografica riscontrabile dopo il Mille in tutta l'Europa ebbe anche in Sardegna i suoi effetti. Fu messo in atto un processo di nuova colonizzazione delle zone costiere, di quelle collinose e pianeggianti, abbandonate in epoca tardo bizantina e alto-giudicale a causa delle invasioni arabe. Un processo lungo e complesso sul quale le fonti medievali non raccontano molto. A questo inducono, come ha sostenuto John Day: "La distribuzione delle prime concessioni fatte dai giudici sardi alla Chiesa romana di villaggi, di uomini e di bestiame fa pensare ad un movimento di ritorno verso le terre a vocazione cerealicola dalle alture pastorali dell'interno" ${ }^{4}$. A conferma lo studioso ricorda la dislocazione delle nuove diocesi create tra l'XI e il XII secolo, prevalentemente situate in zone pianeggianti ed in minima parte in zone pastorali del nord dell'isola. Si trattò dunque di un movimento di colonizzazione rurale, incentivato nei quattro regni isolani dai sovrani giudicali, sia sulle proprie terre che su quelle del fisco, e dai grandi proprietari laici ed ecclesiastici. Nuove terre che venivano date ai coloni in cambio di prestazioni e della corresponsione di diritti diversi. Un movimento di riconquista «che con il continuo aumento della popolazione si tradusse in una tendenza accentuata alla dispersione dell'abitato all'interno del territorion'.

Questo passaggio di territori favorì la messa a coltura di estese aree incolte. Un'opera di dissodamento che investì le campagne nella quale

'M. M. Bazana. Arabi e Sardi nel Medioevo, Cagliari, 1988, pp. 19-20

'Su questi aspetti ctr. B. Fols, Territorio e paesaggio agrario nella Sardegna medievale. Pisil. 1990

JJ. DAY, Uomini e terre nelle Sardegna coloniale. Torino, 1987, pp. 127-139: IDEM. Alle origini della povertà rurale, in "Le opere e i giorni". a cura di F. Manconi-G. Angioni. Cinisello Balsamo, 1982, pp. 13-32. pp. 193-226; su questi temi e sulle teorie del Day sono di grande interesse le riflessioni di M. TANGheroni. Medioevo firrenico, Pisa, 1992. pp. 44-63

5J. DaY. La Sardegna soto la dominazione pisano-genorese. in "Storia ditalia". vol. X, pp. $21-27$ 
ebbero un ruolo determinante le donazioni dei giudici e dei grandi proprietari dell'aristocrazia giudicale a Enti ecclesiastici e ai monaci. Furono soprattutto Benedettini e Cistercensi, chiamati espressamente affinché «ordinent et lavorent et edificent et plantent" che con la loro opera sottrassero al bosco, alle paludi e alla macchia le grandi distese nei salti ${ }^{6}$.

Le vaste curtes donate comprendevano, oltre a servi e bestiame, seminativi, vigneti e frutteti, molte delle aree incolte e boschive, i saltus e le silve $e^{7}$. I non numerosi registri monastici rimasti, i condaghi, unitamente al tenore delle numerose donazioni effettuate tra XI e XIII secolo, permettono un'analisi delle proprietà monastiche ed ecclesiastiche $\mathrm{e}$ forniscono un quadro sulla loro composizione e sull'uso del bosco, sebbene non consentano di determinare il rapporto tra terre coltivate, incolto e bosco. Si trattava di proprietà molto ampie (il $40 \%$ delle complessive estensioni dei quattro regni giudicali, secondo i calcoli di alcuni studiosi) comprendendo anche aree montuose e boschive, situate in aree periferiche rispetto alla montagna, anche se fino alla montagna si incuneavano ${ }^{8}$.

Nei documenti di fondazione dei monasteri e nelle donazioni che vanno accrescendo via via il patrimonio ecclesiastico il bosco, la silva, trova quasi sempre il suo spazio. Ed è questo tra l'altro l'unico termine che le fonti di ambito giudicale utilizzano per indicare il bosco. I riferimenti a boschi ghiandiferi e al bestiame che vi veniva allevato brado sono molto frequenti. Così troviamo nel 1185 nella donazione di Barisone I d'Arborea alla chiesa di Santa Maria di Pisa un salto de glandi ${ }^{9}$ o ancora nell'atto di fondazione della chiesa e monastero di santa Maria di Bonarcado la concessione di numerosi salti pro glande e pro pastu ${ }^{10}$. I numerosi riferimenti alla presenza di ghiandiferi in tutti i condaghi testimoniano come l'allevamento brado di maiali fosse una delle attività silvo-pastorali

${ }^{6} \mathrm{P}$. Tola, Codex Diplomaticus Sardiniae, Torino, 1861, vol. I, sec. XI, doc. VII, p. 153. Sull'attività dei Benedettini in Sardegna cfrr. B. Fols, Territorio cit., pp. 87-112 e la bibliografia citata.

7P. Tola, Codex cit., doc.,XVII, pp. 161-162; doc. X, pp. 184-185; doc. XXVII, p. 199 ; doc. XL, pp. 206-207; doc. LI, pp. 213-214; doc. LVI, p. 216; doc. LX, pp. 218-219; doc. LXIV, pp. 220-221; doc. CIII, p. 245; doc. CX, pp. 252-253; doc. CXIII, p. 254; doc CXXIII, pp. 260-261; doc. XLIII, pp. 334-337; doc. L, p. 342.

${ }^{8} \mathrm{~F}$. CHERCHI PABA, Evoluzione storica dell'attività industriale, agricola, caccia e pesca in Sardegna, Cagliari, 1974, vol II, pp. 89 e segg.

'P. Tola, Codex cit., vol. I, t. I, doc. CXIII, p. 254.

${ }^{10} \mathrm{P}$. Tola, Codex cit., vol.I, t. I, doc. XXVI, p. 320. 
maggiormente praticata" ${ }^{11}$. Proventi legati ad attività economiche montane compaiono tra gli introiti anche nelle rendite pisane del XIII e del principio del XIV secolo e nel Componyment catalano-aragonese della metà del XIV secolo, compilato sulla base di un precedente componimento pisano. Nelle curatorie di Gippi e Trexenta il Comune aveva delle curtes vacharum situate «in montibus et locis de pascis silvestris» ${ }^{12}$. Tra i frutti del pascolo brado costituiva una ricorrente voce di introito quella dei porci, riscossa in percentuale sui capi di bestiame come nei territori del Sarrabus, di Colostrai, di Quirra e in Ogliastra dove «est consuetum ... quod si aliquis habens porchos eos micteret tempore glandium in prato dominico pro ipsis impinguandis dominus porchorum ...debet solvere Comuni Pisano cum extrahuntur de ipso prato decimam partem integram pro indiviso porchorum mictendorum in dicto prato ut dictum est ${ }^{13}$. I boschi maggiormente redditizi, sotto questo profilo, erano dunque costituiti da querce che garantivano con i loro frutti cibo sufficiente per l'allevamento suino.

Nei condaghi, oltre ai numerosissimi querceti (kerkedu) che entrano con maggior frequenza nella composizione dei saltus, sono attestati alberi da frutto (fichi, peri, meli, pruni, aranci, cotogni, olivi, castagni, noci) inseriti all'interno di vigneti o in coltivazioni specializzate come quell'ortu de sa castania ricordato nel condaghe di Santa Maria di Bonarcado ${ }^{14}$ o il Monte de nukes e il coniatu de mela nel condaghe di San Pietro di Silki ${ }^{15}$. Ma a dominare sono soprattutto le annotazioni sui roveri, sugheri, roverelle, lecci, frassini, pioppi, olmi, allori, corbezzoli, tamerici, oleastri, salici, palme che

\footnotetext{
"Cfr. E. BESTA, Il condaghe di S. Maria di Bonarcado, ristampa del testo riveduto a cura di Maurizio Virdis, Oristano, 1982, schede 1, pp. 3-6; 34, p. 25; 145, pp. 61-62.

${ }^{12} \mathrm{~S}$. PetrucCI, Al centro della Sardegna: Barbagia e Barbaricini nella prima metà del XIV secolo, in "Sardegna, Mediterraneo e Atlantico tra medioevo ed età moderna", a cura di L. D’Arienzo, Roma, 1993, vol. I, p. 293.

${ }^{13}$ Il brano è in F. ARTIZZU, Rendite pisane nel giudicato di Cagliari agli inizi del sec. XIV, Padova, 1958, p. 41; sullo sfruttamento dei salti ghiandiferi notizie anche alle pp. 36-41; p. 46, f. 18 ; p. 58, f. 33; p. 61, f. 36v; IDEM, Liber Fondachi, pp. 226-227; 270, f. 16; p. 273, f. 19v; P. Bofarull, Colección de documentos inéditos, vol. XI Repartimiento de Cerdeña, Barcelona, 1856 (edizione anastatica Barcelona, Bellaterra, 1975) vol. XI, pp. 810-813; p. 818; pp. 834-835; 849, 852; IDEM, Il registro $n^{\circ} 1352$ dell'Archivio di Stato di Pisa (Opera del Duomo), in “Annali della Facoltà di Magistero dell'Università di Cagliari”, nuova serie, vol. VI, parte II (1982), pp. 10-11.

${ }^{14}$ E. BESTA, Il condaghe di Bonarcado cit., scheda 203, p. 82.

${ }^{15} \mathrm{G}$. Bonazzi, Il Condaghe di S. Pietro di Silki, Sassari, 1979, scheda 290.
} 
si distribuivano nelle diverse aree a seconda delle quote a formare la rigogliosa macchia e il fitto bosco ${ }^{16}$.

La caccia che nel resto dell'Europa costituiva una fonte di sostentamento, durante il medioevo giudicale non poteva essere praticata liberamente e le risorse che da questa provenivano erano sottoposte ad un rigido controllo da parte dei giudici e disciplinate sia nella Carta de Logu di Arborea che negli Statuti comunali. A queste grandi cacce collettive, svolte periodicamente secondo calendari stabiliti, erano obbligati a partecipare sia i liberi che i servi e alla fine della battuta le prede dovevano essere radunate in un luogo preciso dove avveniva la spartizione delle carni e delle pelli: cervi (attestati indirettamente da una fitta serie di toponimi), daini, cinghiali, martore e volpi che spettavano quasi interamente al signore. Insomma si trattava di una prestazione dovuta da parte degli abitanti, non solo ai giudici ma anche ad alcuni funzionari del regno e la non partecipazione o la frode costavano pesanti multe e sanzioni ${ }^{17}$.

Le fonti lasciano intuire la ricchezza del bosco e l'importanza delle zone alberate nell'economia della vita quotidiana del medioevo. La tutela del bosco che interessò come fenomeno generale gran parte dei territori europei ebbe anche in Sardegna particolare attenzione.

Gli statuti cittadini sardi, scarsi numericamente e tardi (i più antichi risalgono alla fine del XIII secolo) non sono in grado di testimoniare l'estensione dei boschi rispetto alla superficie complessiva dei territori di pertinenza e contengono norme dirette a tutelare quelli di uso comune e non delle aree alberate che rientrano nelle proprietà dei privati. Limiti che ciò non ostante li rendono comunque importanti per comprendere la politica dell'Autorità in relazione al patrimonio boschivo. Tre sono le direttive comuni: protezione del bosco dagli incendi, limitazione del taglio degli alberi e garanzia dell'uso dei pascoli.

Il Breve di Iglesias è quello più attento. Dai capitoli di epoca pisana, riconfermati dai catalano-aragonesi nei primi decenni del Trecento, emerge il tentativo del potere pubblico di contenere e disciplinare lo sfruttamento del

${ }^{16} \mathrm{Cfr}$. G. Meloni-A. Fulgheri, Mondo rurale e Sardegna del XII secolo, Napoli, 1994, pp. 180-181, c. 10v; G. BonAzZI, Il condaghe di S. Pietro di Silki cit., schede 10, 13, 19, 62, 96, 145, 186, 187, 191, 192, 202, 203, 206, 220, 221, 228, 275, 290, 297, 303, 311, 312, 344, 349, 356, 381, 398, 401, 420, 423, 430; E. BESTA, Il condaghe cit., schede cit.

${ }^{17} \mathrm{~L}$. D'ARIEnzo, La caccia in Sardegna nel periodo giudicale e pisano-genovese, in "Medioevo. Saggi e Rassegne”, 6 (1981), pp. 27-60; F. CHERCHI PABA, Evoluzione dell'attività cit., pp. 215 e segg. 
bosco. Iglesias era una città mineraria, la città dell'argento, come sottolinea il titolo del bel libro di Marco Tangheroni. Per lo sfruttamento delle vene argentifere e per la lavorazione del prezioso metallo aveva necessità di ingenti quantità di risorse legnose come combustibile. Qui i tagli furono con molte probabilità praticati assai precocemente, sia nelle zone di fitta macchia che in quelle boschive, nelle montagne prossime ai giacimenti ${ }^{18}$. Un processo che come ha rilevato Angela Terrosu Asole, dovette investire da tempi più antichi, non solo i rilievi dell'iglesiente ma anche quelli della Nurra, del Sarrabus e della Barbagia meridionale dove erano concentrate le più importanti risorse metallifere ${ }^{19}$. $\mathrm{E}^{\prime}$ in questo senso - tutela di un'attività economica altamente redditizia - che si incanalano le norme statutarie iglesienti. L'incendio dei boschi è severamente vietato e si impone a coloro che facevano carbone di non procurare danni «ad alcuno bosco che abbisogna nell'argentiera». Il capitolo inserisce una interessante annotazione che specifica quali fossero i boschi vietati nell'iglesiente: «E intendasi che sia bosco vietato, lo quale bosco sia bosco vietato, lo quale bosco sia bosco di ceppi di smarrare, et di pertiche o scaldatoie o da ligname da serrare». Negli altri boschi «possa fare carbone a sua volontà». Risultano quindi comprese all'interno del termine bosco sia la macchia che il bosco vero e proprio con «il ligname da serrare $»^{20}$.

E' sempre nel Breve che si trova l'unico caso di rimboschimenti effettuati man mano che avveniva il taglio degli alberi: nel capitolo LIII gli Statuti fanno esplicito riferimento a «boschi antichi e novelli». Risulta ovvio

\footnotetext{
${ }^{18}$ M. TANGHERONI, La cittì dell'argento, Napoli, 1985, pp. 39-42. A conferma della presenza di boschi nell'iglesiente un documento regio del 1338 concede a coloro che si recano a Villa di Chiesa di poter pascolare i buoi, i cavalli e altri animali nei salti e nelle foreste come "erat assuetum tempore Pisanorum" (C. BAUDI DI VESME, Coder cit., doc. LIV, pp. 419-420).

${ }^{19}$ A. Terrosu Asole, I paesaggi di montagna, in "La Sardegna”, a cura di M Brigaglia, vol. I, La geografia, la storia, l'arte e la letteratura, Sassari, 1982, pp. 43-47.

${ }^{20} \mathrm{C}$. BAudi DI VeSme, Codex Diplomaticus Ecclesiensiens, Torino, 1987, p. 68, cap. LIII; altri capitoli del Breve legati all'uso del legname e del carbone per l'estrazione e per tutelare patrimonio boschivo e coltivazioni: cap. XVIII, p. 96 (Di non mettere fuoco in alcumo boscho); cap. XLVIII, p. 109 (Di fuocho, che non si debbia mettere in boscho); cap. L, p. 110 (Delle persone che non possano comperare alcuno legname da serrare); cap. LXXI, p. 119 (Di non comperare legname per revendere); cap. VIII, p. 191 (Delle montagne, boschi et acque potere beneficiare); cap. LI, p. 211 (Delli molentari che portano il legname); cap. LXVI, p. 222 (Delli mulintari et carraturi, che non gittino la vena et li carboni). Testimonianze dell'attività dei carbonai si trovano per l'area logudorese in G. BONAZZI, Il condaghe cit., schede 145 e 381 e nella curatoria di Gippi dove, intorno al 1359, sono documentati boscaioli -carbonai che "non arant set tamen faciunt carbone et ligna pro vendendo". Cfr. J. DAY, La Sardegna sotto la dominazione pisano-genovese, Torino, 1987, tab. 8 e p. 107.
} 
che $\mathrm{i}$ boschi novelli erano impiantati per non depauperare le risorse in funzione dell'attività mineraria ${ }^{21}$. Ancora nei capitoli, si insiste su come le norme dovessero essere rispettate per «lo meliore statu et accrescimento dell'argentiera" e si stabilisce che da tutti i boschi e salti che sono nel regno di Cagliari, si potesse trarre «ognia legname necessario et bisognevile per beneficiare l'argentiera, cioè ceppi, et scaldatore, et tucto lo legname che bisogna a forni che colino l'ariento e lo ligname da fuoco et da serrare bisognevile alle fosse» senza il pagamento di alcun diritto tranne quello di $\mathbf{s}$. $\mathrm{XV}$ per cenerraccio ${ }^{22}$.

Altre norme del Breve disciplinano gli incendi dei boschi in funzione agricola, vietando il fuoco entro le tre miglia dalla cinta urbana, dal che si ricava che propaggini boschive o di macchia erano comprese entro l'immediato perimetro urbano ${ }^{23}$.

Nella stessa ottica troviamo disciplinati gli incendi e il taglio degli alberi negli Statuti di Sassari e di Castelsardo. Le pene sono molto elevate: a Sassari, in tutti i territori di pertinenza del Comune, era vietato il taglio della legna e l'espianto di alberi di qualunque tipo dietro il pagamento della pena pecuniaria e la riparazione del danno; pena che in caso di insolvibilità pecuniaria del reo era pagata nei casi più gravi con l'impiccagione. L'obiettivo non era solo quello di proteggere i campi e gli orti che circondavano l'immediata cinta urbana e tutelare vigne, olivi e altri alberi da frutto ma in modo esplicito gli stessi boschi considerati un'importante risorsa per le attività economiche ${ }^{24}$.

Anche gli Statuti di Castelsardo della metà del Quattrocento vietavano gli incendi nei boschi non lontano dalla città (in Basalorgia e Rogulana) da metà giugno a metà settembre. Un capitolo (XXV) è dedicato al divieto di «faguer linna segare cum alcunu ferru in Basalorgia» sotto pena di 100 soldi. Prevede però -e qui si rientra negli usi consentiti per il consumo e le attività quotidiane - la raccolta e il taglio di legna secca «pro

\footnotetext{
"Il testo del Breve di Villa di Chiesa ricorda "... tucti li boschi et salti le quali sono in de lo Regno di Callari, anthici et novelli, si possano traggere ognia legname neceessario..." Cfr. C. BAUdi di Vesme, Coder Diplomaticus cit., cap. LIII, p. 68.

2'C. BAUdI dI VESME, Codex Diplomaticus cit., cap. LIII, p. 68.

${ }^{23}$ C. BAUdI DI VESME, Codex Diplomaticus cit., p. 96, cap. XVIII. p. 109, cap. XLVIII: p. 110, cap. L.

${ }^{24}$ P. Tola, Codex Diplomaticus cit., vol. I. t. II, p. 536. cap. XLI (De non secare limna in su monte); cap. XLII (De non ponner focu): cap. LXV (Dessos qui venden su palone et issa limna): p. 590, cap. XXVII (De non bocare arbores).
} 
fauer sa bugada e pro lavare lana» però sença ferru. Evidentemente legna secca e minuta che poteva essere raccolta con le mani senza danneggiare le piante ${ }^{25}$.

Analogamente gli Satuti sassaresi vietano il taglio della legna nelle campagne e nei monti vicini alla città, vietano severamente il taglio e l'espianto di alberi e disciplinano il fuoco per evitare danni ai coltivi ${ }^{26}$.

Più complesse, anche se legate esclusivamente alla protezione dei coltivi, sono le norme della seconda metà Trecento della Carta de Logu di Mariano IV d'Arborea che rientrano nella politica di rilancio delle attività agricole nel giudicato e a questo sono circoscritte ${ }^{27}$. Il sovrano arborense in diversi capitoli disciplina l'uso del fuoco per proteggere i campi coltivati e le vigne e arginare gli sconfinamenti di bestiame. Il fuoco poteva essere appiccato nei campi nei mesi estivi sotto accurato controllo e dietro pene severissime in caso di incendi a vigneti o orti. Un uso, questo del fuoco, tradizionale come pratica agraria della debbiatura, utilizzata anche come rapido mezzo di fertilizzazione del terreno dai pastori ${ }^{28}$.

Eppure anche l'Arborea includeva grandi aree montagnose e boscate, ma non vi sono esplicite misure difensive. Nel 1228, nella donazione di numerose montagne fatta da Pietro II d'Arborea ai Benedettini di San Martino di Oristano, è specificato come nelle montagne concesse con tutti i boschi, terre colte e incolte «stricti e rigorose comandamus in generali non atrevetsi persona nexuna faguer dannu, ne in terras, ne in arbores, ne bruxiare boscu, ne linnayolu nexunu linna tallare ne pasturare né minus narbones bogare, ne lahoru nexunu faguere in terra de ditos montes et saltos sensa lissensia de sos ministradores o procuradores de sa dita Ecclesia, pagande sempre su feudu eo portadiga a sa Ecclesia de Santu Martini de Aristanis»: si tratta di un provvedimento, all'interno della concessione, che non mira a proteggere il bene in quanto tale ma garantire la proprietà e i

\footnotetext{
${ }^{25} \mathrm{E}$. BESTA, Intorno ad alcuni frammenti di un antico statuto di Castelsardo, Modena, 1899 , cap. CCXII, CCXIII (Qui nexiunu pognat fogu), cap. CCXV (Qui nexunu depiat faguer.. alcuna linna segare cum ferru in basalorgia) $\mathrm{pp}$ 47-48.

${ }^{26} \mathrm{P}$. Tola, Codex cit., vol. I, t. II, capp. XLI e XLII, p. 356; cap. XXVII, p. 590.

${ }^{27}$ E. Besta-P. E. Guarnerio, Carta de Logu de Arborea, Sassari, 1905, pp. 52-52: B. Fols, Territorio e paesaggio agrario nella Sardegna medievale, Pisa, 1990, pp. 145-165.

${ }^{28}$ Sull'antica pratica della debbiatura M. LE LanNou, Pastori e contadini di Sardegna, Cagliari, 1979, p. 64
} 
diritti del monastero, limitando la possibilità d'uso dei cittadini arborensi che potevano legnarvi solo dietro il pagamento di un tributo ${ }^{29}$.

I salti e $\mathrm{i}$ boschi demaniali e feudali erano utilizzati anche dalla popolazione rurale addensata nelle ville o sparsa in piccoli gruppi tra colline e montagne per la raccolta della legna, dei frutti, delle erbe. Sui salti e sui boschi gravavano diritti ademprivili che consentivano, nel limite imposto dal soddisfacimento delle necessità personali, la raccolta di legna per gli usi domestici e personali ${ }^{30}$.

Frutti come le ghiande in annate di carestia sostituivano il grano. Il Wagner ricorda come ancora ai primi del Novecento «solo in pochi villaggi dell'isola, tra i più poveri e i più remoti, a Baunei, Triei, Urzulei, Talana, Villagrande Strisaili e nella vallata di Gairo, si prepara ancora un pane di farina di ghiande, cui s'aggiunge una certa quantità d'argilla ricca di ferro per neutralizzare l'acido tannico delle ghiande. La preparazione di questo pane è abbastanza complicata ... Prima si fanno bollire le ghiande nell'acqua calda e quando sono rammollite, si pestano nel mortaio; poi con uno spianatoio rotondo di legno si riducono in una poltiglia, che si stende su una lastra di pietra. Qui la poltiglia si mescola con l'argilla e se ne impastano le focacce, che s'ungono con un pò di grasso di maiale o d'olio, per dar loro sapore, e poi si cospargono con un pò di cenere, per evitare che si attacchino al fondo del forno» ${ }^{31}$. Un pasto poverissimo, ma risorsa vitale offerta nei secoli ancora dal bosco davanti allo spettro della fame .

I cittadini di Cagliari per reperire il legname dovevano allontanarsi dalla città. Secondo antichi privilegi che risalivano all'epoca giudicale ancora nel Trecento e nei secoli successivi, avevano diritto di rifornirsi di legna in gran parte dei territori del cagliaritano. I diritti di ademprivio di cui godevano consentivano loro l'approvvigionamento da Capoterra a Capo

\footnotetext{
29" Et gasi stricti et rigorose comandamus in generali non atrevetsi persona nexuna faguer dannu, nè in terras, nè in arbores, nè bruxare boscu, nè linnayolu nexunu, linna tallare, nè pasturare, nè minus narbones bogare, nè lahoru nexunu faguere in terras de ditos montes, et saltos, sensa lissencia de sos Ministradores, ò procuradores de sa dita Eclesia, pagande sempre su feudu, eo portadiga à sa Eclesia de Santu Martini de Aristanis” in P. TOLA, Codex Diplomaticus cit, vol. I, t. I, doc. XLVII, pp. 340-34.

${ }^{30} \mathrm{Cfr}$. A. Solmi, Ademprivia, Studi sulla proprietà fondiaria in Sardegna, Pisa, 1904; E. GESSA, Una città e il suo territorio forestale. Gli ademprivi di Cagliari nei secoli XIV-XV, in "VII Settimana della cultura scientifica", Sassari, 1997, pp. 106-108.

${ }^{31}$ M. L. WAGNeR, La vita rustica, a cura di G. Paulis, Nuoro, 1996, pp. 168-169.
} 
Carbonara $^{32}$. Non senza resistenza da parte dei feudatari a cui i catalanoaragonesi dopo la conquista avevano infeudato quelle terre. Le periodiche composizioni dimostrano la frequenza delle controversie anche nei secoli successivi. Ai cittadini di Cagliari era concesso fin dal 1331 il privilegio secondo il quale era loro consentito far legna nei boschi del cagliaritano senza il pagamento di alcun diritto sia per le loro necessità «lignam ad usum seu provisionem vite ipsorum" sia "fustam seu lignamina ad opus palissade machinarum sive trabuchs verdescarum poncium januarium et aliorum fornimentorum Castri predicti vel appendiciorum eiusdem ${ }^{33}$.

Di grande interesse è l'accordo stipulato nel 1360 tra la città di Cagliari e Francesch Roig signore delle ville di Sarroch, Garabio, de Petra Sale, de Sancta Maria Magdalena e de Cuccho nel quale oltre a venire confermati i diritti di legnatico dei cagliaritani era concordato che tutti $\mathrm{i}$ catalani o aragonesi sudditi del re, se lo avessero ritenuto opportuno, navigando verso Cagliari o nella costa cagliaritana con le loro imbarcazioni «puxen tallar e boscar e fer tot lenyam e tota fusta e lenya trer dels dits boschs e termens sens pagament de algun dret e per mercaderia e altres tots lurs volontats. Exceptats emperò arbres domestichs qui seran o sien per fer fruyt». Anche per loro vi è l'esenzione dal pagamento dei diritti purché quella legna e quei tronchi siano impiegati per la costruzione di edifici o in usi personali nel Castello o nelle Appendici ${ }^{34}$.

Il problema degli usi civici era sentito con notevoli resistenze anche nel Capo di Logudoro dove nel Quattrocento furono sollevate delle controversie, una delle quali ricordata nel Codice di S. Pietro di Sorres, sul diritto di legnatico dal signore di Cabuabbas. I vassalli chiamati a testimoniare certificarono tutti come durante l'epoca giudicale, sia sotto Brancaleone Doria che il Visconte di Narbona agli abitanti del Meilogu non era mai stato "devedado ligna" e mai era stata chiesta licenza, anzi quelli del Meilogu «andayt ad omni saltu, silva, buschu, per omni lenya ad posta sua, leant lenya de ogni genia» e quanti avevano conosciuto Brancaleone e il

\footnotetext{
${ }^{32} \mathrm{R}$. DI TUCCI, Il Libro Verde della cittì di Cagliari. Cagliari, 1925, doc. XXXXI, pp. 145154; doc. LVI, pp. 174-177; doc. LXIII. pp. 184-185; doc. CXLVIII, pp. 290-292; doc. CCLXIII, pp. 451-453.

${ }^{33}$ R. DI TUCCI, /l Libro cit., doc. LVIII, p. 184 (14 luglio 1331).

${ }^{34} \mathrm{~L}$ accordo è riportato in R. DI TUCCI, Il Libro cit., doc. CXLIII, pp. 290-292.
} 
visconte confermarono come nei decenni precedenti «non fuyt may devedado ligna de domos, fogo ne atera sorte ${ }^{35}$.

Le aree urbane, oltre che per gli usi domestici, come quello essenziale della cucina, necessitavano di consistenti quantità di legname per forni, bagni e per le attività artigianali che vi si svolgevano. Se il legname minuto era facilmente reperibile, grazie ai privilegi di cui gli abitanti godevano, compresi quelli delle ville dei territori infeudati, più complesso era l'approvvigionamento di grossi tronchi, ben documentato per la città di Cagliari. L'Ogliastra, relativamente a Cagliari, viene spesso definita dalle fonti il luogo "on se buscha la fusta ${ }^{36}$. Le folte foreste della Sardegna sud occidentale da dove fin dall'epoca pisana sono attestate consistenti esportazioni di legname sono tra le poche descritte nelle fonti documentarie del Trecento. Nel 1335 il castellano di Quirra lamentava i problemi di sicurezza posti dalle frequenti imboscate tese dai barbaricini che nel fitto della selva trovavano il nascondiglio adatto per tendere agguati ai soldati catalani. Tra il castello di Quirra e Villaputzu, scriveva il castellano, «est quidam passus ad modum periculosus et nocivus propter nemus densum quod ibi est in quo semper continue morantur et stant in aguayte homines malefactores qui sunt de Barbarya», i quali erano reponsabili di numerosi crimini. La soluzione individuata dal sovrano fu quella di bruciare e sradicare dictum nemum, anche se i conflittuali rapporti con i Barbaricini non si risolsero con questo intervento. E la rigogliosità della vegetazione, oggi molto più diradata, poco dovette essere intaccata da un abbattimento per altro improbabile ${ }^{37}$.

Dagli stessi boschi del cagliaritano, come da quelli elencati sotto il controllo di Francesch Roig, si potevano prelevare - e certo lo si era fattotronchi per la palizzata e per opere edili di un certo rilievo ${ }^{38}$. Eppure solo fino alla metà del Trecento si attestano esportazioni di legname dal porto di Cagliari che nel corso del secolo si vanno affievolendo. Durante tutta la prima metà del Trecento troviamo sporadici riferimenti a tagli di legname per la costruzione di remi per le galere della grande cantieristica navale

\footnotetext{
${ }^{35}$ A. Sanna, Il codice S. Pietro di Sorres, Cagliari, 1957. schede 301-305. pp. 126-130.

3"Cir. C. Manca, Il Libro dei conti di Miquel Ça Rovira. Padova. 1969. p. 31.

${ }^{37}$ S. Petrucci, Al centro della Sardegna, cit.. pp. 298-299.

${ }^{38}$ C. Manca. Il Libro dei conti. cit., pp. 30-32: 52-54.
} 
barcellonese ${ }^{39}$. A partire dalla seconda metà del secolo il flusso si inverte e le importazioni di legname sulla piazza cagliaritana appaiono consistenti. Alcune delle cause possono essere ricercate nella guerra con il giudicato di Arborea, che creava difficoltà di comunicazione. Il legname di taglia inferiore risultava ancora disponibile grazie alla possibilità offerte dai boschi del circondario, pur con le periodiche cride che nei momenti di maggior pericolo vietavano ai cittadini di allontanarsi per non cadere nelle imboscate. Per le grandi travature e per interventi di particolare rilievo, come la ristrutturazione della palizzata del porto di Cagliari o la riparazione del centinaio di abitazioni bruciate nell'incendio divampato in città nel 1386, si era costretti a far ricorso al mercato siciliano e ai boschi della costa laziale $^{40}$.

Tavole di abete di Venezia o della Calabria e generiche tavole provenienti da Napoli e dalla Corsica risultano tra le merci in entrata in porto ancora in epoca moderna. E' possibile che la scarsa varietà di essenze rispetto alle necessità o la mancanza di specializzazione dei boschi rendesse meno conveniente la ricerca in loco di grandi quantità di tronchi, tipologicamente omogenei, di cui necessitava il mercato urbano, trovando piuttosto conveniente l'acquisto altrove, dove era più facile reperire legname pregiato e delle dimensioni richieste ${ }^{41}$.

Aldilà del mito di una Sardegna coperta di boschi su cui più volte anche la storiografia attuale si è soffermata, emerge la notevole estensione

\footnotetext{
${ }^{39}$ Pisa percepiva dall'Ogliastra, per l'esportazione di legname "pro quolibet lignamino cuiusque currus videlicet de quo possit fieri unus currus quod inciditur et extrahitur de boschis s. 1. Attestazioni esportazione legname sardo dal porto di Cagliari si hanno ancora verso metà Trecento: tra il 1352 e il 1354 Pietro IV ordina in Sardegna remi e altro legname per la flotta navale in allestimento per la spedizione nell'isola. Archivo de la Corona de Aragón, Cancilleria, reg. 1397 , cc. 189-191; reg. 1400 , cc. 5, 52, 56, 68-70; 76v.

${ }^{40}$ Nelle Ordinazioni dei Consiglieri di Cagliari del XIV secolo è stabilita la tarifta che barcaioli possono riscuotere per scaricare tavole di abete di Venezia o della Calabria e generiche tavole di Napoli ed un apposito capitolo vietava l'esportazione di legname per mare (M. PInNa, Le Ordinazioni dei Consiglieri del Castello di Cagliari, Cagliari, 1927, cap. 94, p. 49; cap. 119, pp. 119-211); nel 1390 in seguito all'incendio che aveva devastato numerose case in Castello si importa legname dalla Sicilia. Il legname fu acquistato dal mercante barcellonese Pere Pertegaç il quale fu attaccato in mare mentre rientrava. Nella costa laziale fu acquistato il legname per riattare le torri e per la palizzata del porto di Cagliari, cfir. C. MANCA, Il Libro dei conti cit., pp. 31-33, 216-218.

${ }^{4}$ Anche le dogane attestano importazione di legname da Venezia, Napoli, Calabria e Corsica dal XIV secolo. Nel corso del Cinquecento dagli statuti portuali di Cagliari risulta inportata grande varietà di legname sotto forma di tronchi e tavole e di arredi. Cfr. P. F. SimBULA, Gli statuti doganali del porto di Cagliari (in preparazione).
} 
del manto arboreo testimoniata ancora fino al secolo scorso. Gli stessi di provvedimenti in materia di tutela del patrimonio boschivo, concentrati nell'area di pertinenza delle città di Sassari e di Castelsardo inducono a pensare ad un sostanziale un equilibrio tra uomo e ambiente vegetale; interventi legati prevalentemente a contenere un impoverimento delle risorse da parte delle comunità rurali e urbane quando ancora tra medioevo e età moderna i disboscamenti e l'attività dei carbonai erano dirette a soddisfare le necessità interne e non quelle del mercato esterno. Quando ancora i «parecchi boschi ombrosi e splendide selve fitte di alberi d'alto fusto» da cui «si ricava legname adatto alla costruzione di case, navi ed altri utensili» ricordati dal Fara alla fine del Cinquecento erano erti nella maestosità delle loro chiome ancora lontani per qualche secolo dalle scuri che si sarebbero abbattute nell'Ottocento ${ }^{42}$.

\section{RÉSUMÉ}

La croissance démographique au début du nouveau millénaire produit même en Sardaigne un procès de colonisation des nouveaux territoires et des changements dans les installations. L'expansion agricole et l'exploitation des terrains incultes et des bois a été observé en regardant les plus anciennes témoignages du moyen âge, les "condaghi”, les textes statutaires du bas moyen âge et les sources littéraires. En considérant les essences les plus répandues dans l'île, ce travail analyse les tapes de l'exploitation du bois pour la culture. l'élevage, la chasse et le bois de charpente. en regardant la sauvegarde de cette importante ressource. Par les statuts et les textes normatifs a été reconstruite la politique de discipline d'exploitation du bois et le procès de reboisement au XIV" siècle.

\section{SUMMARY}

The population growth at the beginning of the new millennium determined even in Sardinia the colonisation of new territories and changes in the settlement. In this work the expansion of the agriculture as well as the exploitation of the woody-land have been studied by examining the "condaghi", the more ancient testimonies of Middle Ages, the late medieval statutory texts, and the literary sources. This work considers the most diffused crops in the

\footnotetext{
${ }^{42}$ I. F. FARA, Opera. In Sardiniae Chorographiam, cit., pp. 110-111. Sulla mediocrità della foresta sarda già prima dell'Ottocento insiste M. LE LANNOU, Pastori e contadini cit., pp. 6172; in contrasto M. BRIGAGLIA, Il paesaggio agrario, in "Le opere e i giorni" cit., pp. 191193.
} 
island and analyses the growing of the woodland exploitation for cultivation, breeding, hunting and building-timber. as well as the protection of this resource. The policy of regulating the exploitation of the woody-land and the reforesting process which started in the XIV century have been studied by analysing the normative texts and the civic statutes. 